\title{
ON STABILITY OF DELAYED DIFFERENTIAL SYSTEMS OF ARBITRARY NON-INTEGER ORDER
}

\author{
TOMÁŠ KISELA
}

\begin{abstract}
This paper summarizes and extends known results on qualitative behavior of solutions of autonomous fractional differential systems with a time delay. It utilizes two most common definitions of fractional derivative, Riemann-Liouville and Caputo one, for which optimal stability conditions are formulated via position of eigenvalues in the complex plane. Our approach covers differential systems of any non-integer orders of the derivative. The differences in stability and asymptotic properties of solutions induced by the type of derivative are pointed out as well.
\end{abstract}

\section{INTRODUCTION}

In many areas of science and technology we often meet problems which are well described by differential systems with a time delay. Examples of such situations might be reaction time of technical and chemical systems or heredity in population dynamics. Qualitative theory for these equations is summarized in, e.g. [2,5]. The study of delayed systems involving viscoelasticity, anomalous diffusion or control theory naturally suggests to enrich our models with derivatives of non-integer order which proved to be very effective in these areas (see, e.g. $[4,8]$ ).

This is the main motivation for our study of two delayed systems which can be written as

$$
\begin{aligned}
& \mathbf{D}_{0}^{\alpha} y(t)=A y(t-\tau), \quad t \in(0, \infty), \alpha \in \mathbb{R}^{+} \backslash \mathbb{Z}, \\
& y(t)=\phi(t), \quad t \in[-\tau, 0], \\
& \left.\mathbf{D}_{0}^{\alpha-k} y(t)\right|_{t=0}=y_{\alpha-k}, \quad k=1, \ldots,\lceil\alpha\rceil
\end{aligned}
$$

and

$$
\begin{aligned}
& { }^{C} \mathrm{D}_{0}^{\alpha} y(t)=A y(t-\tau), \quad t \in(0, \infty), \alpha \in \mathbb{R}^{+} \backslash \mathbb{Z}, \\
& y(t)=\phi(t), \quad t \in[-\tau, 0], \\
& y^{(\lceil\alpha\rceil-k)}(0)=y_{\lceil\alpha\rceil-k}, \quad k=1, \ldots,\lceil\alpha\rceil,
\end{aligned}
$$

where $\mathbf{D}_{0}^{\alpha}$ and ${ }^{C} \mathrm{D}_{0}^{\alpha}$ denote the so-called Riemann-Liouville and Caputo differential operators of order $\alpha$, respectively. Further, $A \in \mathbb{R}^{d \times d}$ is a constant $d \times d$ matrix, $y . \in \mathbb{R}^{d}$ are constant vectors and $\tau>0$ is a constant delay. As usual for delayed

$M S C$ (2010): primary 34K37, 34K20; secondary 34K25.

Keywords: fractional delay differential system, stability, asymptotic behavior, RiemannLiouville derivative, Caputo derivative.

The research was supported by the grant GA20-11846S of the Czech Science Foundation. 
equations, the initial condition is given by $\phi \in L^{1}[-\tau, 0]$ (componentwise) and the use of fractional derivatives allows us to prescribe also initial values for $t=0$ separately. We intentionally leave out the integer-order values of $\alpha$ since they coincide with the known classical cases.

A serious qualitative analysis of such equations is being performed less than two decades. It spans across scalar and vector cases, various methods like $D$ decomposition or Laplace transform are used. For more details we refer to $[1,3,6,9]$ which are the main sources for this paper.

The paper is organized as follows. In Section 2 we outline some basic preliminary results useful in our further considerations. Section 3 is devoted to the discussion of solution representations and their comparison. The main results are concentrated in Section 4 where we summarize known facts as well as derive some original ones. Section 5 concludes the paper with a few final remarks.

\section{Preliminaries}

Let $f$ be a real function. We use the standard definition of fractional integral of order $\gamma>0$

$$
\mathrm{I}_{0}^{\gamma} f(t)=\int_{0}^{t} \frac{(t-\xi)^{\gamma-1}}{\Gamma(\gamma)} f(\xi) \mathrm{d} \xi, \quad t \geq 0 .
$$

We employ both the wide used definitions of fractional derivative of order $\alpha>0$ called the Riemann-Liouville and Caputo derivative introduced as

$$
\begin{aligned}
& \mathbf{D}_{0}^{\alpha} f(t)=\frac{\mathrm{d}^{\lceil\alpha\rceil}}{\mathrm{d} t^{\lceil\alpha\rceil}}\left(\mathrm{I}_{0}^{\lceil\alpha\rceil-\alpha} f(t)\right), \quad t \geq 0, \\
& { }^{C} \mathrm{D}_{0}^{\alpha} f(t)=\mathrm{I}_{0}^{\lceil\alpha\rceil-\alpha}\left(\frac{\mathrm{d}^{\lceil\alpha\rceil}}{\mathrm{d} t^{\lceil\alpha\rceil}} f(t)\right), \quad t \geq 0,
\end{aligned}
$$

respectively. Additionally, we put ${ }^{C} \mathrm{D}_{0}^{0} f(t)=\mathbf{D}_{0}^{0} f(t)=f(t)$ (for more information on fractional operators we refer, e.g. to $[4,8])$.

The key tool, utilized throughout this paper, is the Laplace transform which is, for $f$, introduced as

$$
\mathcal{L}(f(t))(s)=\int_{0}^{\infty} \exp \{-s t\} f(t) \mathrm{d} t, \quad s \in \mathbb{C}
$$

provided the integral converges. To perform the transform of (1.1) and (1.4), we need a clear view on Laplace transform of a function with shifted (delayed) argument which is given by

$$
\begin{aligned}
& \mathcal{L}(f(t-\tau) h(t-\tau))(s)=\exp \{-\tau s\} \mathcal{L}(f(t))(s), \quad \tau>0 \\
& \mathcal{L}(f(t-\tau))(s)=\exp \{-\tau s\} \mathcal{L}(f(t))(s)+\exp \{-\tau s\} \int_{-\tau}^{0} \exp \{-s t\} f(t) \mathrm{d} t, \quad \tau>0 .
\end{aligned}
$$


Also, using the formulae for Laplace transform of convolution and power function

$$
\begin{aligned}
& \mathcal{L}\left(\int_{0}^{t} f(t-\xi) g(\xi) \mathrm{d} \xi\right)(s)=\mathcal{L}(f(t))(s) \cdot \mathcal{L}(g(t))(s), \\
& \mathcal{L}\left(\frac{t^{\eta}}{\Gamma(\eta+1)}\right)(s)=s^{-\eta-1}, \quad \eta>-1
\end{aligned}
$$

we can see the origin of Laplace transforms of fractional operators

$$
\begin{aligned}
& \mathcal{L}\left(\mathrm{I}_{0}^{\gamma} f(t)\right)(s)=s^{-\gamma} \mathcal{L}(f(t))(s), \quad \gamma>0, \\
& \mathcal{L}\left(\mathbf{D}_{0}^{\alpha} f(t)\right)(s)=s^{\alpha} \mathcal{L}(f(t))(s)-\left.\sum_{k=1}^{\lceil\alpha\rceil} s^{k-1} \mathbf{D}_{0}^{\alpha-k} f(t)\right|_{t=0}, \quad \alpha>0, \\
& \mathcal{L}\left({ }^{C} \mathrm{D}_{0}^{\alpha} f(t)\right)(s)=s^{\alpha} \mathcal{L}(f(t))(s)-\sum_{k=1}^{\lceil\alpha\rceil} s^{\alpha-k} f^{(k-1)}(0), \quad \alpha>0 .
\end{aligned}
$$

The symbol $h$ denotes the Heaviside step function defined as $h(\xi)=1$ for $\xi \geq 0$ and $h(\xi)=0$ for $\xi<0$. When applied on a vector function, the Laplace transform is considered componentwise.

We note that the system matrix $A$ of (1.1) and (1.4) can be rewritten with the use of a matrix $\Lambda$ in a Jordan canonical form with the Jordan blocks on its diagonal as $A=T \Lambda T^{-1}$, where $T$ is a regular real $d \times d$ matrix,

$$
\Lambda=\left(\begin{array}{cccc}
J_{1} & 0 & \cdots & 0 \\
0 & J_{2} & \cdots & 0 \\
\vdots & \vdots & \ddots & \vdots \\
0 & 0 & \cdots & J_{q}
\end{array}\right), \quad J_{k}=\left(\begin{array}{ccccc}
\lambda_{i} & 1 & 0 & \cdots & 0 \\
0 & \lambda_{i} & 1 & \ddots & \vdots \\
\vdots & \ddots & \ddots & \ddots & 0 \\
0 & \cdots & & \lambda_{i} & 1 \\
0 & \cdots & & 0 & \lambda_{i}
\end{array}\right), \quad k=1, \ldots, q
$$

and $\lambda_{i}(i=1, \ldots, n)$ are distinct eigenvalues of $A$. The number of Jordan blocks corresponding to $\lambda_{i}$ is called geometric multiplicity of $\lambda_{i}$. The sum of the sizes of all Jordan blocks corresponding to $\lambda_{i}$ is called algebraic multiplicity of $\lambda_{i}$.

Before we proceed to the next section, we recall the stability notions related to our linear fractional differential systems with a delay. The zero solution is said to be stable (asymptotically stable) if the solution of the system is bounded (tends to zero as $t \rightarrow \infty)$ for any initial function $\phi \in L^{1}([-\tau, 0])$.

\section{Solution Representations For (1.1) And (1.4)}

As in the integer-order case (see, e.g. [2,5]), an essential role is played by analogue of the fundamental matrix solution also for (1.1) and (1.4) (see, e.g. [1]). In order to simplify the notation dealing with the orders $\alpha$ greater than one, we introduce its generalization in form of the following functions

$$
R_{\alpha, \beta}^{A, \tau}(t)=\mathcal{L}^{-1}\left(\left(s^{\alpha} I-A \exp \{-s \tau\}\right)^{-1} s^{\alpha-\beta}\right)(t), \quad \alpha \in \mathbb{R}^{+} \backslash \mathbb{Z}, \beta \in \mathbb{R}^{+}
$$

where $A \in \mathbb{R}^{d \times d}$ and $I$ is the identity $d \times d$ matrix. Employing these $R$-functions, we arrive at the following solution representations. 
Theorem 3.1. The solution $y_{R L}$ of (1.1)-(1.3) is given by

$$
y_{R L}(t)=\sum_{k=1}^{\lceil\alpha\rceil} R_{\alpha, \alpha-k+1}^{A, \tau}(t) y_{\alpha-k}+\int_{-\tau}^{0} R_{\alpha, \alpha}^{A, \tau}(t-\tau-u) A \phi(u) \mathrm{d} u .
$$

Proof. Applying (2.1), (2.2) on (1.1)-(1.3), we get

$$
\begin{aligned}
& \mathcal{L}(y(t))(s) \\
& =\left(s^{\alpha} I-A \exp \{-s \tau\}\right)^{-1}\left[\sum_{k=1}^{\lceil\alpha\rceil} s^{k-1} y_{\alpha-k}+\int_{-\tau}^{0} \exp \{-s(t+\tau)\} A \phi(t) \mathrm{d} t\right] \\
& =\sum_{k=1}^{\lceil\alpha\rceil} \mathcal{L}\left(R_{\alpha, \alpha-k+1}^{A, \tau}(t)\right)(s) y_{\alpha-k}+\int_{-\tau}^{0} \exp \{-s(t+\tau)\} \mathcal{L}\left(R_{\alpha, \alpha}^{A, \tau}(t)\right)(s) A \phi(t) \mathrm{d} t
\end{aligned}
$$

which yields the assertion.

Theorem 3.2. The solution $y_{C}$ of (1.4)-(1.6) is given by

$$
y_{C}(t)=\sum_{k=1}^{\lceil\alpha\rceil} R_{\alpha, k}^{A, \tau}(t) y_{k-1}+\int_{-\tau}^{0} R_{\alpha, \alpha}^{A, \tau}(t-\tau-u) A \phi(u) \mathrm{d} u .
$$

Proof. Analogously as above, applying (2.1), (2.3) on (1.4)-(1.6), we obtain

$$
\begin{aligned}
& \mathcal{L}(y(t))(s) \\
& =\left(s^{\alpha} I-A \exp \{-s \tau\}\right)^{-1}\left[\sum_{k=1}^{\lceil\alpha\rceil} s^{\alpha-k} y_{k-1}+\int_{-\tau}^{0} \exp \{-s(t+\tau)\} A \phi(t) \mathrm{d} t\right] \\
& =\sum_{k=1}^{\lceil\alpha\rceil} \mathcal{L}\left(R_{\alpha, k}^{A, \tau}(t)\right)(s) y_{k-1}+\int_{-\tau}^{0} \exp \{-s(t+\tau)\} \mathcal{L}\left(R_{\alpha, \alpha}^{A, \tau}(t)\right)(s) A \phi(t) \mathrm{d} t
\end{aligned}
$$

which again concludes the proof.

Remark 3.3. We can see that the integral terms involving the initial function $\phi$ are for $y_{R L}$ and $y_{C}$ identical. The difference occurs in the terms involving the local initial conditions. Although the Caputo case is more studied in the literature, in particular of order $\alpha \in(0,1]$ (see, e.g. $[1,3,6]$ ), the Riemann-Liouville one actually appears to be structurally closer to the classical case. Indeed, $R_{\alpha, \alpha}^{A, \tau}$ seems to be playing practically the same role as the fundamental matrix solution in integerorder delay differential equations.

It might look like Theorems 3.1 and 3.2 are not that much explicit since the $R$-functions are defined via the inverse Laplace transform. Now we show that these functions can be actually evaluated pretty straighforwardly.

Applying the Jordan canonical form theory, we can write $\mathcal{L}\left(R_{\alpha, \beta}^{A, \tau}(t)\right)(s)=\left(s^{\alpha} I-A \exp \{-s \tau\}\right)^{-1} s^{\alpha-\beta}=T\left(s^{\alpha} I-\Lambda \exp \{-s \tau\}\right)^{-1} s^{\alpha-\beta} T^{-1}$. 
Clearly, the matrix $\left(s^{\alpha} I-\Lambda \exp \{-s \tau\}\right)^{-1} s^{\alpha-\beta}$ is block diagonal with the blocks given by upper triangular strip matrices of the form

$$
\left(s^{\alpha} I-J_{k} \mathrm{e}^{-s \tau}\right)^{-1} s^{\alpha-\beta}=\left(\begin{array}{cccc}
\frac{s^{\alpha-\beta}}{s^{\alpha}-\lambda_{i} \mathrm{e}^{-s \tau}} & \frac{\mathrm{e}^{-s \tau} s^{\alpha-\beta}}{\left(s^{\alpha}-\lambda_{i} \mathrm{e}^{-s \tau}\right)^{2}} & \cdots & \frac{\mathrm{e}^{-(r-1) s \tau} s^{\alpha-\beta}}{\left(s^{\alpha}-\lambda_{i} \mathrm{e}^{-s \tau}\right)^{r k}} \\
0 & \frac{s^{\alpha-\beta}}{s^{\alpha}-\lambda_{i} \mathrm{e}^{-s \tau}} & \ddots & \frac{\mathrm{e}^{-(r-2) s \tau} s^{\alpha-\beta}}{\left(s^{\alpha}-\lambda_{i} \mathrm{e}^{-s \tau}\right)^{r} k^{-1}} \\
\vdots & \vdots & \ddots & \vdots \\
0 & 0 & \cdots & \frac{s^{\alpha-\beta}}{s^{\alpha}-\lambda_{i} \mathrm{e}^{-s \tau}}
\end{array}\right)
$$

where $J_{k}(k=1, \ldots, q)$ is the $k$-th block of $\Lambda$ and $r_{k}$ is its size. It was proven in [1] that the elements of this matrix can be expressed as

$$
\frac{\exp \{-m s \tau\} s^{\alpha-\beta}}{\left(s^{\alpha}-\lambda \exp \{-s \tau\}\right)^{m+1}}=\mathcal{L}\left(G_{\alpha, \beta}^{\lambda, \tau, m}(t)\right)(s)
$$

where

$$
G_{\alpha, \beta}^{\lambda, \tau, m}(t)=\sum_{j=0}^{\lceil t / \tau-m-1\rceil}\left(\begin{array}{c}
m+j \\
j
\end{array}\right) \frac{\lambda^{j}(t-(m+j) \tau)^{\alpha(m+j)+\beta-1}}{\Gamma(\alpha(m+j)+\beta)}, \quad t>0 .
$$

To summarize the previous considerations, we can write the following assertion.

Lemma 3.4. Let $A \in \mathbb{R}^{d \times d}, \lambda_{i}(i=1, \ldots, n)$ be distinct eigenvalues of $A$ and let $p_{i}$ be the largest size of the Jordan block corresponding to the eigenvalue $\lambda_{i}$. Then the non-zero elements of matrix function $R_{\alpha, \beta}^{A, \tau}$ are linear combinations of scalar functions

$$
G_{\alpha, \beta}^{\lambda_{i}, \tau, m}(t), \quad m=0, \ldots, p_{i}-1, \quad i=1, \ldots, n .
$$

\section{MAin RESUlts}

It is well known from the basic theory of the Laplace transform method that if all poles of the Laplace image of solutions (roots of the so-called characteristic equation) have negative real parts, then the zero solution of the studied equation is asymptotically stable (and their non-zero solutions tend to zero in an exponential rate). On the other hand, if there exists a pole with a positive real part, the corresponding zero solution is not stable (its absolute value tends to infinity exponentially). In the fractional case, it usually occurs a more complex situation, involving also singular points and poles with the zero real parts, which require a deeper analysis.

For our fractional problems (1.1) and (1.4), as it can be seen from the proof of Theorems 3.1 and 3.2, the characteristic equation takes the form

$$
\operatorname{det}\left(s^{\alpha} I-A \exp \{-s \tau\}\right)=0 \quad \text { or } \quad \prod_{i=1}^{n}\left(s^{\alpha}-\lambda_{i} \exp \{-s \tau\}\right)^{w_{i}}=0,
$$

where $\lambda_{i}(i=1, \ldots, n)$ are distinct eigenvalues of $A$ and $w_{i}$ are the corresponding algebraic multiplicities. As we can see from (4.1) and (3.1), for further eigenvalues considerations it is sufficient to investigate the roots of the equation

$$
p(s ; \lambda) \equiv s^{\alpha}-\lambda \exp \{-s \tau\}=0
$$


where $\lambda$ is a complex parameter. Now, we perform a direct root analysis of (4.2). In particular, we formulate the optimal conditions on $\lambda$ ensuring that (4.2) does not have any root with positive real part.

Lemma 4.1. Let $\alpha \in \mathbb{R}^{+} \backslash \mathbb{Z}, \tau>0$ and $\lambda \in \mathbb{C}$. Then all the roots of (4.2) have negative real parts if and only if

$$
\alpha \in(0,2), \quad|\operatorname{Arg}(\lambda)|>\frac{\alpha \pi}{2} \quad \text { and } \quad|\lambda|<\left(\frac{|\operatorname{Arg}(\lambda)|-\alpha \pi / 2}{\tau}\right)^{\alpha}
$$

where $\operatorname{Arg}(\lambda) \in(-\pi, \pi]$ is the principal argument of $\lambda$.

Proof. The case $\lambda=0$ is trivial since then (4.2) has only the zero solution which does not satisfy (4.3). Let $\lambda \neq 0$ and put

$$
s=r \exp \{\mathrm{i} \varphi\}, \quad \lambda=\varrho \exp \{\mathrm{i} \psi\}
$$

where $r=|s|, \varrho=|\lambda|$ and $\varphi, \psi \in(-\pi, \pi]$ are principal arguments of $s, \lambda$, respectively. Then we can write (4.2) for real and imaginary parts as a system of two equations in the form

$$
\begin{gathered}
r^{\alpha} \cos (\alpha \varphi)-\varrho \exp \{-r \tau \cos (\varphi)\} \cos (\psi-r \tau \sin (\varphi))=0, \\
r^{\alpha} \sin (\alpha \varphi)-\varrho \exp \{-r \tau \cos (\varphi)\} \sin (\psi-r \tau \sin (\varphi))=0 .
\end{gathered}
$$

Now, let us assume that (4.2) has a root with a non-negative real part, i.e. $|\varphi| \leq \pi / 2$.

For $\varphi=0$, we have $\psi=0$ (i.e. $\lambda=\varrho$ ) from (4.5). Further, (4.4) implies, for $r$ and $\varrho$, the relation $r^{\alpha}=\varrho \exp \{-r \tau\}$ which always allows to find an appropriate $r$ to a given $\varrho$. Hence, (4.2) has a non-negative real root if and only if $\lambda$ is a nonnegative real.

Let $|\varphi| \in(0, \pi / 2] \backslash\{\pi / \alpha\}$. Since $|\varphi| \neq \pi / \alpha$, we have $\psi-r \tau \sin (\varphi) \neq k \pi$ for any $k \in \mathbb{Z}$ and, by dividing and rearranging (4.4) and (4.5), we arrive at a new reformulation of (4.4), (4.5) in the form

$$
\begin{aligned}
\alpha \varphi & =\psi-r \tau \sin (\varphi)+2 k \pi, \\
r^{\alpha} & =\varrho \exp \{-r \tau \cos (\varphi)\}
\end{aligned}
$$

for a suitable $k \in \mathbb{Z}$ (the replacement of $k \pi$ by $2 k \pi$ is implied by positivity of $r$ and $\varrho$ ). Further, by eliminating $r$ from (4.6), (4.7), we get the equation for $\varphi$ as

$$
\left(\frac{\psi-\alpha \varphi+2 k \pi}{\tau \sin (\varphi)}\right)^{\alpha}=\varrho \exp \{(\alpha \varphi-\psi-2 k \pi) \cot (\varphi)\}
$$

As proven in [1] for $\alpha \in(0,1)$, the left-hand side is decreasing with respect to $\varphi$ on $(0, \pi / 2]$ with the lowest value at $\varphi=\pi / 2$ for any $k$. The right-hand side is increasing with respect to $\varphi$ on $(0, \pi / 2]$ with the largest value at $\varphi=\pi / 2$ for any $k$. It can be easily checked that the situation for $\alpha \geq 1$ is the same provided we put the left-hand side equal to zero for $\varphi$ such that $\psi-\alpha \varphi+2 k \pi<0$. Obviously, the existence of a root $\varphi \in(0, \pi / 2]$ for at least one $k$ is ensured if and only if

$$
|\psi| \leq \frac{\alpha \pi}{2} \quad \text { or } \quad \varrho \geq\left(\frac{|\psi|-\alpha \pi / 2}{\tau}\right)^{\alpha} .
$$


We can see that $(4.8)_{1}$ is automatically satisfied for $\alpha \geq 2$, hence for $\alpha \geq 2$ there is always a root of (4.2) with a non-negative real part. We can see that, for $\alpha \in(0,2)$, (4.8) is a complement of (4.3).

So far, we have not investigated the situation $|\varphi|=\pi / \alpha \leq \pi / 2$. However, it can occur only for $\alpha \geq 2$ and in that case we already know that there is always a root of (4.2) with a non-negative real part.

Summarizing the previous arguments, we can conclude the proof.

Lemma 3.4 shows that functions of the type $G_{\alpha, \beta}^{\lambda, \tau}$ play, for (1.1) and (1.4), an analogous role as exponential functions for integer-order systems. Hence, it is crucial to have a good uderstanding of asymptotic behavior of $G_{\alpha, \beta}^{\lambda, \tau, m}$ and its relation to (4.2) which is provided by the following assertion which slightly extends the result presented in [1].

Lemma 4.2. Let $\lambda \in \mathbb{C}, \alpha, \beta, \tau \in \mathbb{R}^{+}$and $m \in \mathbb{Z}$ be such that $\alpha \in \mathbb{R}^{+} \backslash \mathbb{Z}$, $m \geq 0$. Further, let $s_{i}(i=1,2, \ldots)$ be the roots of (4.2) with ordering $\Re\left(s_{i}\right) \geq$ $\Re\left(s_{i+1}\right)$ (in particular, $s_{1}$ is the zero with the largest real part).

(i) If $\lambda=0$, then

$$
G_{\alpha, \beta}^{0, \tau, m}(t)=\frac{(t-m \tau)^{m \alpha+\beta-1}}{\Gamma(m \alpha+\beta)} h(t-m \tau) .
$$

(ii) If $\lambda$ is such that $s_{1}$ has negative real part, then

$$
\begin{aligned}
G_{\alpha, \beta}^{\lambda, \tau}(t)= & \frac{(-1)^{m+1}}{\lambda^{m+1} \Gamma(\beta-\alpha)}(t+\tau)^{\beta-\alpha-1} \\
& +\frac{(-1)^{m+1}(m+1)}{\lambda^{m+2} \Gamma(\beta-2 \alpha)}(t+2 \tau)^{\beta-2 \alpha-1}+\mathcal{O}\left(t^{\beta-3 \alpha-1}\right) \quad \text { as } t \rightarrow \infty .
\end{aligned}
$$

(iii) If $\lambda$ is such that $s_{1}$ is purely imaginary or it has positive real part, then

$$
\begin{aligned}
G_{\alpha, \beta}^{\lambda, \tau, m}(t)= & \sum_{j=0}^{m}(t-m \tau)^{j}\left(a_{j} \exp \left\{s_{1}(t-m \tau)\right\}\right. \\
& \left.+b_{j} \exp \left\{s_{2}(t-m \tau)\right\}\right)+\left\{\begin{array}{cl}
\mathcal{O}\left(t^{m} \exp \left\{\Re\left(s_{3}\right) t\right\}\right), & \text { if } \Re\left(s_{3}\right) \geq 0, \\
\mathcal{O}\left(t^{\beta-\alpha-1}\right), & \text { if } \Re\left(s_{3}\right)<0
\end{array}\right. \\
& \text { as } t \rightarrow \infty
\end{aligned}
$$

where $a_{j}, b_{j}$ are suitable nonzero complex constants $(j=0, \ldots, m)$.

Proof. The assertion was proved in [1] for the case $\alpha \in(0,1)$. The generalization for $\alpha>1$ is a tedious but direct analogue.

Now we are in a position to formulate the main results of this paper. For the sake of lucidity, we introduce the following subset of complex numbers motivated by $(4.3)$ as

$$
\mathcal{S}_{\alpha, \tau}=\left\{\lambda \in \mathbb{C}:|\lambda|<\left(\frac{|\operatorname{Arg}(\lambda)|-\alpha \pi / 2}{\tau}\right)^{\alpha},|\operatorname{Arg}(\lambda)|>\frac{\alpha \pi}{2}\right\},
$$

which we call the stability region of (1.1) and (1.4). 
Theorem 4.3. Let $A \in \mathbb{R}^{d \times d}, \alpha \in \mathbb{R}^{+} \backslash \mathbb{Z}$ and $\tau>0$. Further, let $p_{0} \in \mathbb{Z}$ be the largest size of the Jordan block corresponding to the zero eigenvalue of $A$, where we put $p_{0}=0$ if $A$ has only non-zero eigenvalues.

(i) The zero solution of (1.1) is asymptotically stable if and only if $\alpha \in(0,2)$, all non-zero eigenvalues of $A$ belong to $\mathcal{S}_{\alpha, \tau}$ and $p_{0}<1 / \alpha$.

(ii) The zero solution of (1.1) is stable if and only if $\alpha \in(0,2)$, all eigenvalues of $A$ belong to $\mathrm{cl}\left(\mathcal{S}_{\alpha, \tau}\right)$, all non-zero eigenvalues of $A$ lying on $\partial \mathcal{S}_{\alpha, \tau}$ have the same algebraic and geometric multiplicities and $p_{0} \leq 1 / \alpha$.

Proof. Theorem 3.1 and Lemma 3.4 imply that the solution components of (1.1) are formed as linear combinations of functions

$$
G_{\alpha, \alpha-k+1}^{\lambda_{i}, \tau, m}(t) \text { and } \int_{-\tau}^{0} G_{\alpha, \alpha}^{\lambda_{i}, \tau, m}(t-\tau-u) \phi_{j}(u) \mathrm{d} u
$$

where $k=1, \ldots,\lceil\alpha\rceil, \lambda_{i}(i=1, \ldots, n)$ are eigenvalues of $A, m$ is a non-negative integer as specified in Lemma 3.4 and $\phi_{j}(j=1, \ldots, d)$ are components of the initial function.

Lemma 4.1 implies that all roots of (4.1) have negative real part if and only if $\alpha \in(0,2)$ and all eigenvalues belong to $\mathcal{S}_{\alpha, \tau}$. Moreover, (4.1) has at least one root with zero real part and other roots with a negative real part if and only if at least one eigenvalue lies on the boundary of $\mathcal{S}_{\alpha, \tau}$.

Thus, the asymptotic behavior of the solution can be derived from Lemma 4.2. The functions $(4.9)_{1}$ are described directly, we just point out that for $\lambda_{i} \in \mathcal{S}_{\alpha, \tau}$ the first term in the expansion cancels out due to the negative integer argument in the Gamma function, so that we obtain

$$
G_{\alpha, \alpha-k+1}^{\lambda_{i}, \tau, m}(t)=\frac{(-1)^{m+1}(m+1)}{\lambda_{i}^{m+2} \Gamma(-\alpha-k+1)}(t+2 \tau)^{-\alpha-k}+\mathcal{O}\left(t^{-2 \alpha-k}\right) \quad \text { as } t \rightarrow \infty .
$$

Now, we investigate $(4.9)_{2}$. Employing the assuption $\phi \in L^{1}[-\tau, 0]$ and Lemma 4.2, we can distinguish several cases:

Let $\alpha \in(0,2)$ and $\lambda_{i} \in \mathcal{S}_{\alpha, \tau}$. The second mean value theorem implies

$$
\begin{aligned}
& \int_{-\tau}^{0} G_{\alpha, \alpha}^{\lambda_{i}, \tau, m}(t-\tau-u) \phi(u) \mathrm{d} u=G_{\alpha, \alpha}^{\lambda_{i}, \tau, m}(t) \int_{-\tau}^{\xi} \phi(u) \mathrm{d} u \\
& =K_{1}(t+2 \tau)^{-\alpha-1}+\mathcal{O}\left(t^{-2 \alpha-1}\right) \quad \text { as } t \rightarrow \infty,
\end{aligned}
$$

where $K_{1} \in \mathbb{R}$ is non-zero and $\xi \in(-\tau, 0]$.

Now, let $\lambda_{i}=0$. By the same approach we arrive at

$$
\int_{-\tau}^{0} G_{\alpha, \alpha}^{0, \tau, m}(t-\tau-u) \phi(u) \mathrm{d} u=K_{2}(t-m \tau)^{(m+1) \alpha-1},
$$

where $K_{2} \in \mathbb{R}$ is non-zero. This expression vanishes for $t \rightarrow \infty$, if and only if $m+1=p_{0}<1 / \alpha$.

The cases for $\lambda_{i} \in \partial \mathcal{S}_{\alpha, \tau} \backslash\{0\}$ and $\lambda_{i} \notin \mathrm{cl}\left(\mathcal{S}_{\alpha, \tau}\right)$ can be handled similarly. We arrive at the conclusion that $(4.9)_{2}$ is bounded, when the non-zero eigenvalue lying on the boundary of stability region has the same algebraic and geometric multiplicity. Otherwise the absolute value of $(4.9)_{2}$ increases polynomially (when the eigenvalue lies on the boundary) or exponentially. 
Theorem 4.4. Let $A \in \mathbb{R}^{d \times d}, \alpha \in \mathbb{R}^{+} \backslash \mathbb{Z}$ and $\tau>0$. Further, let $p_{0} \in \mathbb{Z}$ be the largest size of the Jordan block corresponding to the zero eigenvalue of $A$, where we put $p_{0}=0$ if $A$ has only non-zero eigenvalues.

(i) The zero solution of (1.4) is asymptotically stable if and only if $\alpha \in(0,2)$ and all eigenvalues of $A$ belong to $\mathcal{S}_{\alpha, \tau}$.

(ii) The zero solution of (1.4) is stable if and only if $\alpha \in(0,2]$, all eigenvalues of $A$ belong to $\operatorname{cl}\left(\mathcal{S}_{\alpha, \tau}\right)$, all non-zero eigenvalues of $A$ lying on $\partial \mathcal{S}_{\alpha, \tau}$ have the same algebraic and geometric multiplicities and $p_{0} \leq 2-\lceil\alpha\rceil$.

Proof. The idea of the proof is equivalent to that one of Theorem 4.3. In particular, the solution components of (1.4) are given by linear combinations of

$$
G_{\alpha, k}^{\lambda_{i}, \tau, m}(t) \text { and } \int_{-\tau}^{0} G_{\alpha, \alpha}^{\lambda_{i}, \tau, m}(t-\tau-u) \phi_{j}(u) \mathrm{d} u,
$$

where $k=1, \ldots,\lceil\alpha\rceil, \lambda_{i}(i=1, \ldots, n)$ are eigenvalues of $A, m$ is a non-negative integer as specified in Lemma 3.4 and $\phi_{j}(j=1, \ldots, d)$ is a component of the initial function. Thus, we see that $(4.10)_{2}$ is the same as $(4.9)_{2}$ while $(4.10)_{1}$ differs with respect to $(4.9)_{1}$ due to the change of index. This causes only a different decay rate for $\lambda_{i} \in \operatorname{cl}\left(\mathcal{S}_{\alpha, \tau}\right)$.

Overall, there is only one difference in stability behavior which occurs for $\lambda_{i}=0$ when we have

$$
G_{\alpha, k}^{0, \tau, m}(t)=\frac{(t-m \tau)^{m \alpha+k-1}}{\Gamma(m \alpha+k)} .
$$

We can see that this function never tends to zero with $t \rightarrow \infty$ and it is bounded if and only if $m \alpha+k-1=0$ which means $\lceil\alpha\rceil=1$ (i.e. $k=1$ ) and $p_{0}=1$ (i.e. $m=0)$.

Remark 4.5. (i) Theorems 4.3 and 4.4 show that $\mathcal{S}_{\alpha, \tau}$ is the stability region for delayed fractional differential systems for Riemann-Liouville and Caputo derivative, i.e. for (1.1) and (1.4), respectively. Figure 1 represents the situation for $\alpha \in(0,1)$ when the stability region includes also points with positive real part. We can see in Figure 2 how the region is transformed for $\alpha \in(1,2)$, and it is apparent how the stability region vanishes for $\alpha \rightarrow 2$. Also, for $\tau \rightarrow 0, \mathcal{S}_{\alpha, \tau}$ tends to the stability region known from theory of fractional differential equations without delay (see, e.g. $[7,9]$ ).

(ii) From the stability viewpoint, the only difference between (1.1) and (1.4) occurs if there is a zero eigenvalue and the order of derivatives is less than 1 . In this case, the zero solution to (1.1) can be asymptotically stable, stable or unstable, depending on the particular value of $\alpha$ and multiplicities of the zero eigenvalue. The zero solution of (1.4) is stable if algebraic and geometric multiplicities of the zero eigenvalue are equal, otherwise it is unstable (i.e. it does not depend on the particular value of $\alpha$ ).

The proof technique used for Theorems 4.3 and 4.4 actually reveals more than the stability properties. Due to its constructive nature we can actually derive also the asymptotic behavior of the solutions to (1.1) and (1.4). We summarize the comparisons of the two cases in the following assertions dealing with the asymptotic 


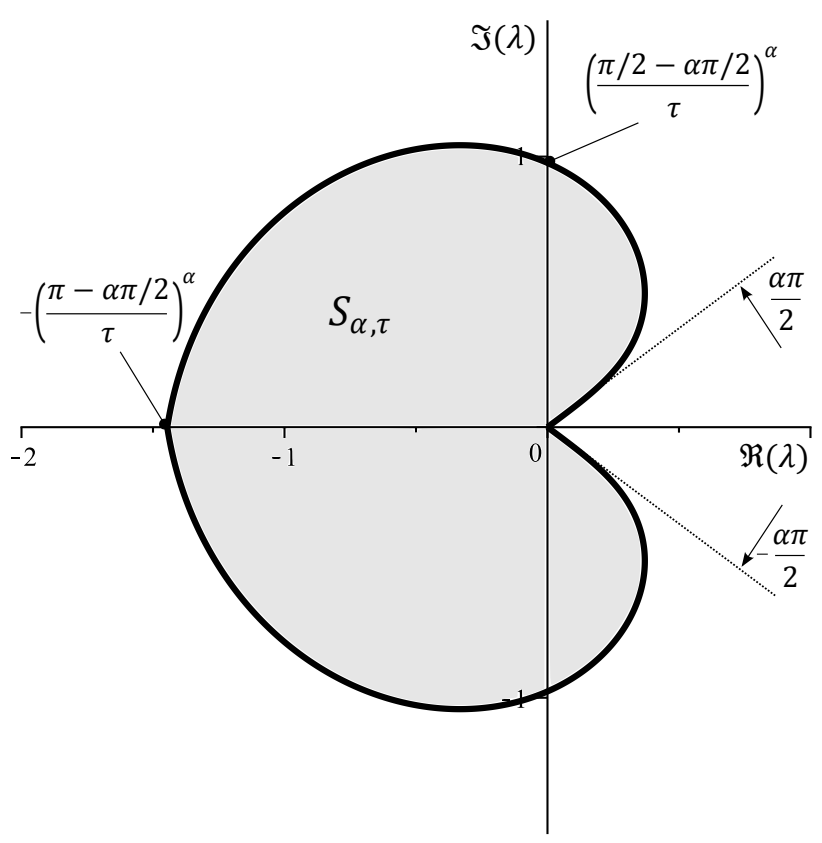

Figure 1. The stability region $\mathcal{S}_{\alpha, \tau}$ for the values $\alpha=0.4$ and $\tau=1$.

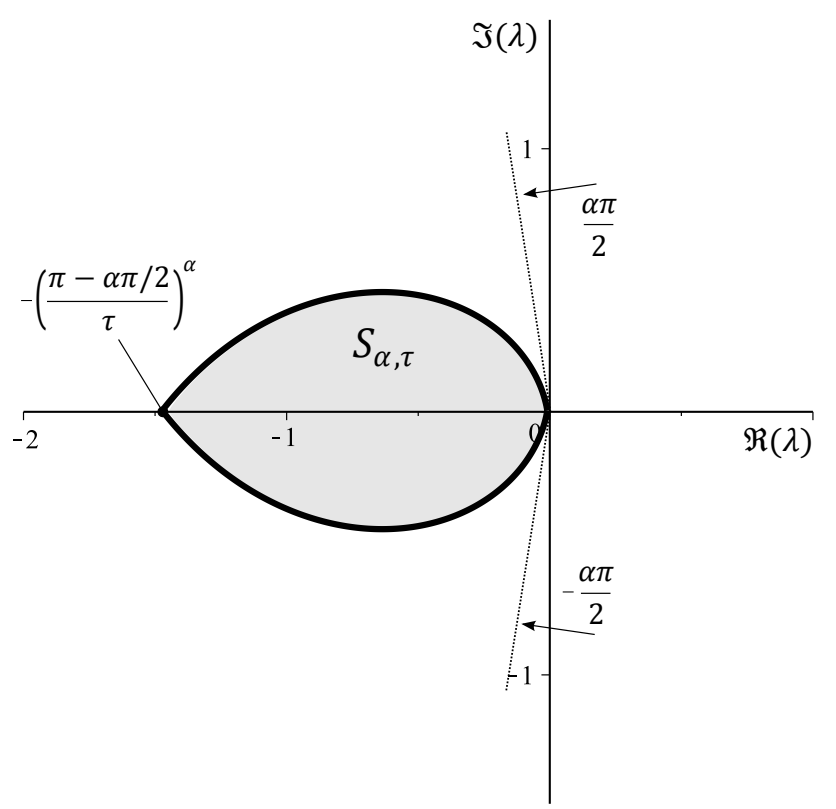

Figure 2. The stability region $\mathcal{S}_{\alpha, \tau}$ for the values $\alpha=1.1$ and $\tau=1$. 
equivalence (denoted by the symbol $\sim$ ) relationships for norms of solutions (we use the symbol $\|\cdot\|$ for Euclidean norm in $\mathbb{R}^{d}$ ).

Theorem 4.6. Let $A \in \mathbb{R}^{d \times d}, \alpha \in(0,2), \tau>0$ and let all the eigenvalues of $A$ belong to $\mathcal{S}_{\alpha, \tau}$. Further, we denote by $y_{R L}$ and $y_{C}$ the solutions of (1.1)-(1.3) and (1.4)-(1.6), respectively. Then it holds

$$
\left\|y_{R L}(t)\right\| \sim t^{-\alpha-1} \quad \text { and } \quad\left\|y_{C}(t)\right\| \sim t^{\lceil\alpha\rceil-\alpha-1} \quad \text { as } t \rightarrow \infty
$$

for almost all choices of initial conditions. If $y_{R L}$ and $y_{C}$ do not follow (4.11), then their norms tend to zero with a faster decay rate.

Proof. Theorems 3.1 and 3.2 indicate some particular choices of initial conditions, e.g. $y_{0}=0$, which can remove the dominating terms from $y_{R L}$ and $y_{C}$ and therefore affect the decay rate. The particular asymptotic properties are then implied by Lemma 4.2 .

Theorem 4.7. Let $A \in \mathbb{R}^{d \times d}, \alpha \in(0,2)$ and $\tau>0$. Let $A$ has the zero eigenvalue and denote $p_{0}$ the size of the largest Jordan block corresponding to this zero eigenvalue. Let all non-zero eigenvalues of $A$ belong to $\mathcal{S}_{\alpha, \tau}$. Further, we denote $y_{R L}$ and $y_{C}$ the solutions of (1.1)-(1.3) and (1.4)-(1.6), respectively. Then it holds

$$
\left\|y_{R L}(t)\right\| \sim t^{p_{0} \alpha-1} \quad \text { and } \quad\left\|y_{C}(t)\right\| \sim t^{\left(p_{0}-1\right) \alpha+\lceil\alpha\rceil-1} \quad \text { as } t \rightarrow \infty
$$

for almost all choices of initial conditions. If $y_{R L}$ and $y_{C}$ do not follow (4.12), then their norms are even smaller for $t$ large enough.

Proof. The idea of the proof is analogous to the previous case.

Remark 4.8. (i) We can observe an interesting distinction between the way how the asymptotic behavior of $y_{R L}$ and $y_{C}$ depends on $\alpha$. While in the RiemannLiouville case we see the algebraic decay rate depending directly on $\alpha$, in the Caputo case the decay rate is driven by the decimal part of $\alpha$, i.e. by the difference $\lceil\alpha\rceil-\alpha$. Indeed, if we consider for example $\alpha_{1}=0.4$ and $\alpha_{2}=1.4$, then the solutions of (1.4) follow essentially the same asymptotic relations, while the Riemann-Liouville ones do not.

(ii) We can employ a similar analysis also in the cases that are not covered by Theorems 4.6 and 4.7, i.e. when there is a non-zero eigenvalue on the boundary or outside the closure of the stability region. We note that if there is a non-zero eigenvalue lying outside the closure of the stability region, the norms of non-zero solutions increase exponentially for both (1.1) and (1.4).

(iii) We point out that the asymptotic results obtained for the delayed fractional differential systems actually mirror the well-known results for fractional differential systems without a delay.

\section{Conclusions}

We have summarized and extended the results on qualitative behavior of solutions of delayed fractional differential systems (1.1) and (1.4) of arbitrary order.

We have shown that the stability of the zero solution occurs only if the order of derivatives is less than 2. Further, we have derived the precise description of 
the stability region which is for both (1.1) and (1.4) identical. The only difference regarding the stability occurs when the system matrix $A$ has a zero eigenvalue. Then we observe the asymptotic stability property for (1.1) only if $\alpha<1$ and the maximum size of the Jordan block corresponding to the zero eigenvalue being less than $1 / \alpha$. In the Caputo case (1.4), the asymptotic stability does not appear and the zero solution is stable (but not asymptotically stable) only if $\alpha<1$ and algebraic multiplicity of the zero eigenvalue being equal to the geometric one.

The asymptotic behavior displays more diversity. If the system matrix $A$ has all eigenvalues lying in the stability region, i.e. the zero solutions of both (1.1) and (1.4) are asymptotically stable, we can generally say that the solutions of (1.1) go to zero as $t \rightarrow \infty$ faster that solutions of (1.4). Moreover, unlike the RiemannLiouville case, the decay rate of solutions to (1.4) does not depend on the value $\alpha$ itself, but on its decimal part only.

The area of qualitative analysis of fractional differential equations with a time delay, especially with higher-order derivative, provides a lot of open problems. Our research may serve as one of the prerequisites to studies of more complex systems, such as $\mathbf{D}_{0}^{\alpha} y(t)=a y(t)+b y(t-\tau)$ or its vector analogues.

\section{REFERENCES}

[1] J. Čermák, J. Horníček and T. Kisela, Stability regions for fractional differential systems with a time delay, Communications in Nonlinear Science and Numerical Simulation 31 (2016), 108-123.

[2] J. K. Hale and S. M. Verduyn Lunel, Introduction to Functional Differential Equations, Springer-Verlag, New York, 1993.

[3] E. Kaslik and S. Sivasundaram, Analytical and numerical methods for the stability analysis of linear fractional delay differential equations, Journal of Computational and Applied Mathematics 236 (2012), 4027-4041.

[4] A. A. Kilbas, H. M. Srivastava and J. J. Trujillo, Theory and Applications of Fractional Differential Equations, Elsevier, Amsterdam, 2006.

[5] V. Kolmanovskii and A. Myshkis, Introduction to the Theory and Applications of Functional Differential Equations, Kluwer Academic Publishers, Dordrecht, 1999.

[6] K. Krol, Asymptotic properties of fractional delay differential equations, Applied Mathematics and Computation 218 (2011), 1515-1532.

[7] D. Matignon, Stability results on fractional differential equations with applications to control processing, in: Computational Engineering in Systems Applications, vol. 2, IMACS, IEEESMC, Lille, 1996, pp. 963-968.

[8] I. Podlubný, Fractional Differential Equations, Academic Press, San Diego, 1999.

[9] D. Qian, C. Li, R. P. Agarwal and P. J. Y. Wong, Stability analysis of fractional differential systems with Riemann-Liouville derivative, Mathematical and Computer Modelling 52 (2010), 862-874.

Tomáš Kisela, Institute of Mathematics, Brno University of Technology, Technická 2, Brno 616 69, Czechia

e-mail: kisela@fme.vutbr.cz 